\title{
Resolução anafórica em português europeu L2: efeitos de animacidade e a posição do antecedente $^{1}$
}

\author{
Joana Teixeira*, Alexandra Fiéis ${ }^{* *}$ e Ana Madeira ${ }^{* *}$ \\ "FLUP \& CLUNL \\ ${ }^{* *}$ NOVA FCSH e CLUNL
}

\begin{abstract}
This study investigates the interpretation of subject pronouns in L2 EP by Italian native speakers, to examine the following questions: In overt subject resolution, do L1 Italian - L2 European Portuguese learners behave like L1 EP speakers regarding antecedent animacy (a property at the syntax-semantics interface) at L2 developmental stages and at the near-native level?; When the antecedent in object position is animate, do L1 Italian - L2 EP learners exhibit permanent optionality in the interpretation of overt subject pronouns but not of null subjects, as claimed by Sorace (2016), a.o.? Participants were 15 adult EP native speakers, 10 intermediate, 10 advanced and 10 near-native Italian adult learners of L2 EP. They were administered two multiple-choice tasks (speeded and untimed) with a 2x2 design crossing the following variables: animacy of the matrix object (animate vs. inanimate) and type of embedded pronominal subject (overt vs. null). Results indicate that L2 learners show problems only in the areas where the L1 and the L2 differ (Madeira, Fiéis \& Teixeira, this volume), namely: the resolution of overt subjects in the presence of [-animate] object antecedent and the resolution of null subjects. Learners' performance in these areas remains unstable even at the near-native level. These findings challenge the ideas that internal interfaces (syntax/semantics) are not persistently problematic and that null subjects are unproblematic in L2 anaphora resolution (cf. Sorace, 2011, 2016). They moreover point to the importance of L1 influence in L2 anaphora resolution, a factor generally played down in previous studies (e.g., Sorace, 2016).
\end{abstract}

Keywords: L2 acquisition, anaphora resolution, animacy, position of the antecedent, European Portuguese

Palavras-chave: aquisição de L2, resolução anafórica, animacidade, posição do antecedente, português europeu

\section{Introdução}

Nas línguas românicas de sujeito nulo (LRSN), os sujeitos pronominais plenos tendem a ocorrer em contextos de mudança de tópico discursivo, recuperando antecedentes não sujeito, enquanto os sujeitos nulos estão associados à manutenção de tópico, retomando antecedentes sujeito (Carminati, 2002). Há evidência de que a interpretação de sujeitos plenos poderá ser sensível não só a estes fatores sintático-discursivos, mas também a um fator semântico como a animacidade do antecedente (Cardinaletti \& Starke, 1999; Morgado et al, 2018). No domínio de aquisição de língua não materna (L2), os estudos sobre resolução anafórica, em regra, têm tido como foco as condições sintático-discursivas que determinam a divisão de trabalho entre sujeitos pronominais nulos e plenos, ignorando o fator animacidade. Muitos destes estudos têm mostrado que os aprendentes de L2 geralmente exibem opcionalidade persistente relativamente a sujeitos plenos, mas não a nulos, independentemente da língua materna (L1) (e.g., Sorace, 2016). Isto tem sido interpretado como

\footnotetext{
${ }^{1}$ Este trabalho foi financiado pela FCT, através do Projeto Estratégico do Centro de Linguística da Universidade Nova de Lisboa UID/LIN/03213/2019. Agradecemos a todos os informantes que participaram no estudo, à Bruna Bragança, à Vera Fernandes e à Maria Luz pela recolha e tratamento dos dados, e a todos os que, de alguma forma, contribuíram para a realização deste estudo.
} 
evidência a favor da Hipótese de Interface (HI) (Sorace \& Filiaci, 2006; Sorace, 2011), segundo a qual as propriedades sintáticas ou envolvendo interfaces internas (e.g., sintaxe-semântica) não são problemáticas em L2, ao passo que as propriedades em interfaces externas (e.g., sintaxe-discurso) são áreas de opcionalidade permanente, mesmo quando a L1 e a L2 são semelhantes. Alguns estudos sobre resolução anafórica (e.g., Rothman, 2009) têm, contudo, produzido resultados que não são consistentes com esta proposta de que as propriedades na interface sintaxe-discurso são permanentemente problemáticas em L2, uma vez que mostram que os aprendentes podem convergir totalmente com a língua alvo na resolução de sujeitos pronominais plenos. Assim, permanece por esclarecer se a resolução de pronomes plenos é necessariamente uma área de permanente opcionalidade em L2, e se aprendentes (muito) avançados de L2 exibem um comportamento alvo em relação a um fator semântico como a animacidade, como predito pela HI. Também a proposta da HI de que os problemas na interface sintaxe-discurso ocorrem mesmo quando a L1 e a L2 são semelhantes carece de confirmação, visto que se baseia em investigação sobre resolução anafórica em pares de línguas de sujeito nulo, em que se ignora a eventual existência de microvariação nestas línguas.

Procurando contribuir para um melhor entendimento destas questões, o presente estudo investiga a interpretação de sujeitos pronominais plenos e nulos em português europeu (PE) L2 em contextos intrafrásicos, considerando os efeitos da posição e da animacidade do antecedente. Os participantes são falantes adultos de italiano L1 nos níveis intermédio, avançado e quase nativo de PE L2. A combinação italiana L1 - PE L2 foi escolhida, por um lado, porque há ainda pouca investigação sobre pares de LRSN e, por outro, porque o PE e o italiano diferem em relação a certos aspetos da resolução anafórica de sujeitos nulos e plenos, o que nos permitirá perceber se os aprendentes de L2 são sensíveis a microvariação neste domínio.

O artigo está estruturado do seguinte modo: a secção 2 apresenta uma visão panorâmica dos estudos prévios sobre resolução anafórica em LRSN como L1 e L2; na secção 3, formulamos as questões de investigação e as predições; a secção 4 explica a metodologia do estudo; os seus resultados são descritos na secção 5; e, finalmente, na secção 6, discutimos os resultados e apresentamos as principais conclusões do estudo.

\section{Estudos prévios sobre resolução anafórica}

\subsection{Resolução anafórica em línguas de sujeito nulo}

Nas LRSN, a divisão de trabalho entre sujeitos pronominais plenos e nulos é determinada por fatores sintáticos e discursivos (e.g., Montalbetti, 1984; Carminati, 2002; Alonso-Ovalle et al., 2002; Luegi, 2012; Lobo, Madeira \& Silva, 2017). Os sujeitos pronominais plenos tendem a retomar antecedentes não sujeito, podendo ser o objeto ou outro referente extralinguístico (1). Discursivamente, estes pronomes estão associados a contextos de mudança de tópico. Já os sujeitos pronominais nulos retomam antecedentes mais proeminentes sintaticamente, nomeadamente, antecedentes em posição de sujeito, em contextos de manutenção de tópico discursivo (2). Segundo Carminati (2002), estas preferências interpretativas correspondem a uma estratégia de parsing, designada como a Estratégia da Posição do Antecedente (Position of Antecedent Strategy).

(1) A mãe dá um beijo à filhaj enquanto ela $\mathrm{j}_{\mathrm{j} / \mathrm{k}}$ veste o casaco.

(2) A mãe dá um beijo à filha $\mathrm{j}_{\mathrm{j}}$ enquanto pro $_{\mathrm{i}}$ veste o casaco.

Há indícios de que um fator semântico como a animacidade do antecedente poderá ser igualmente relevante para a interpretação dos sujeitos plenos. Alguns estudos (Cardinaletti \& Starke, 1999; Barbosa, Duarte, \& Kato, 2005; Morgado et al, 2018) têm mostrado que, na presença de dois potenciais antecedentes, o pronome pleno tende a recuperar o objeto apenas se este for animado. No entanto, não é ainda claro o papel que 
a animacidade desempenha na interpretação de pronomes em LRSN, já que a maioria dos estudos sobre resolução anafórica tem utilizado apenas antecedentes animados.

Com o objetivo de investigar o papel deste fator, Madeira, Fiéis e Teixeira (neste volume) elaboraram um estudo para tentar perceber como os falantes nativos de italiano e de PE interpretam sujeitos pronominais nulos e plenos, manipulando a animacidade dos antecedentes. Este estudo, que tem o mesmo desenho experimental do presente trabalho (ver secção 4.2), mostra que, quando todos os antecedentes potenciais são animados, as duas línguas apresentam preferências idênticas na resolução de sujeitos pronominais plenos, uma vez que em ambas o sujeito pleno retoma preferencialmente o objeto. Porém, quando o antecedente na posição de sujeito é animado e o objeto não é animado, as preferências são diferentes em PE e em italiano: em PE, o sujeito pleno retoma o objeto, e, em italiano, retoma o sujeito. Estes resultados indicam que, em PE, a posição do antecedente é um fator mais relevante na resolução de sujeitos pronominais plenos do que a animacidade. Já em italiano é a animacidade o fator mais preponderante, traduzindo-se na preferência pela retoma de antecedentes animados com sujeitos plenos. Assim, há microvariação nestas LRSN.

Da mesma forma que existe variação quanto ao papel da animacidade na resolução anafórica, também a Estratégia da Posição do Antecedente parece estar sujeita a variação. Num estudo comparativo entre o espanhol e o italiano, Filiaci (2010) e Filiaci, Sorace e Carreiras (2014) mostram que, em frases complexas em que todos os potenciais antecedentes são animados, quando o contexto pragmático força a escolha do antecedente sujeito, os sujeitos pronominais plenos retomam mais facilmente esse antecedente em espanhol do que em italiano. $\mathrm{Na}$ resolução de sujeitos nulos, não se observam diferenças nas duas línguas, pelo que os autores propõem que o sujeito pronominal pleno está mais sujeito a variação interlinguística. Contrariando esta ideia, Madeira, Fiéis e Teixeira (neste volume), utilizando contextos pragmaticamente neutros, atestam a existência de variação na interpretação de sujeitos nulos em PE e em italiano: enquanto, em PE, o sujeito nulo retoma o antecedente na posição de sujeito, em italiano, não há uma preferência clara pela retoma do sujeito ou do objeto, quando todos os potenciais antecedentes são animados (em linha com os resultados do grupo de controlo de Sorace e Filiaci, 2006).

Em suma, os estudos realizados até ao momento indicam que as duas LRSN consideradas no presente estudo, PE e italiano, apresentam diferenças na resolução de sujeitos plenos na presença de objetos não animados e na resolução de sujeitos nulos, tendo preferências idênticas apenas na interpretação de sujeitos plenos na presença de antecedentes animados.

\subsection{Resolução anafórica em aquisição de L2}

No domínio de aquisição de L2, os estudos sobre resolução anafórica têm tido como foco a Estratégia da Posição do Antecedente, considerando apenas contextos em que todos os potenciais antecedentes são animados. De um modo geral, estes estudos têm mostrado que os aprendentes não têm problemas com os sujeitos nulos, mas exibem opcionalidade com os plenos até mesmo no nível quase nativo (e.g., Lozano, 2003; Sorace, 2016; Sorace \& Filiaci, 2006). Esta assimetria é encontrada não só em combinações de línguas em que a L1 é uma língua de sujeito obrigatório e a L2 uma língua de sujeito nulo (e.g., Belletti, Bennati, \& Sorace, 2007; Lozano, 2003, 2006; Sorace \& Filiaci, 2006), como também em pares de línguas em que a L1 e a L2 são línguas de sujeito nulo consistente (e.g., Bini, 1993; Lozano, 2008; Margaza \& Bel, 2006; Mendes \& Iribarren, 2007), o que tem sido interpretado como evidência de que as dificuldades na interface sintaxe-discurso não resultam da influência da L1. Note-se, contudo, que estes estudos com duas línguas de sujeito nulo não têm considerado possíveis efeitos de microvariação.

Em conjunto, estes resultados sobre resolução anafórica em L2 têm sido usados como argumento em favor da Hipótese de Interface (HI) (cf. Sorace, 2011; Sorace \& Filiaci, 2006). Segundo a atual versão desta hipótese (Sorace, 2011), enquanto as propriedades que são estritamente sintáticas ou envolvem interfaces internas (e.g., 
sintaxe-semântica) não são problemáticas em L2, as que envolvem interfaces externas (e.g., sintaxe-discurso) são áreas de opcionalidade permanente. A HI propõe que esta opcionalidade é fruto de ineficiências na integração de informação sintática com informação pragmático-discursiva no uso da língua em tempo real, as quais, por sua vez, são um efeito secundário do bilinguismo (para detalhes, ver Sorace, 2011, 2016). Esta hipótese prediz, pois, que a interface sintaxe-discurso deverá ser uma área de opcionalidade permanente independentemente da combinação L1-L2.

Confirmando a diferença entre interfaces internas e externas predita pela HI, os poucos estudos que testaram, simultaneamente, a aquisição de propriedades nestes dois tipos de interfaces pelos mesmos grupos de falantes (e.g., Serratrice et al., 2009; Sorace \& Serratrice, 2009; Sorace et al., 2009; Tsimpli \& Sorace, 2006) mostraram que, enquanto fenómenos que envolvem interfaces externas geram dificuldades persistentes, interfaces internas como a interface sintaxe-semântica não são problemáticas em níveis quase nativos.

Apesar de a HI ser confirmada por vários estudos, alguns trabalhos apresentam resultados que colocam em causa as suas predições. Por exemplo, Rothman (2009) mostra que, em inglês L1 - espanhol L2, não se verificam problemas na resolução de sujeitos pronominais plenos em níveis mais avançados de proficiência, o que indica que propriedades na interface sintaxe-discurso não são necessariamente problemáticas. Por outro lado, também há evidência de que, em alguns casos, as interfaces internas (e.g., Hopp, 2007; Kraš, 2011) podem gerar dificuldades a falantes quase nativos.

Face ao exposto, algumas questões estão ainda em aberto: (i) se, como defendem alguns estudos anteriores (e.g., Lozano, 2003; Sorace, 2016; Sorace \& Filiaci, 2006), a resolução anafórica de pronomes plenos é necessariamente uma área de permanente opcionalidade em L2; (ii) se é possível que os aprendentes de níveis mais avançados alcancem um conhecimento alvo de um fator semântico como a animacidade (fator do domínio da gramática que não deveria ser problemático), como predito pela HI; e (iii) se os aprendentes são sensíveis à microvariação em línguas de sujeito nulo.

Para tentar responder a estas questões, elaborámos um estudo que tem por objetivo investigar como os falantes de italiano L1 aprendentes de PE L2 interpretam sujeitos pronominais nulos e plenos em frases complexas, manipulando a animacidade dos antecedentes e procurando perceber o papel desempenhado pela L1 na resolução anafórica de duas LRSN.

\section{Questões de investigação}

Tendo em conta o estado da arte apresentado na secção 2, e, em particular, as questões deixadas em aberto em trabalhos anteriores, o nosso estudo sobre resolução anafórica nas gramáticas de falantes de italiano L1 PE L2 procurará investigar as seguintes questões:

QI.1 - Na resolução de sujeitos pronominais plenos, os aprendentes de italiano L1 - PE L2 têm um desempenho semelhante ao dos falantes nativos de PE no que diz respeito à animacidade do antecedente em diferentes estádios de desenvolvimento?

QI.2 - Quando o antecedente na posição de objeto é animado, os aprendentes de italiano L1 - PE L2 exibem opcionalidade permanente na interpretação de pronomes plenos, mas não na de nulos, conforme proposto por Sorace (2016), e.o.?

Apesar das diferenças que se observam entre o italiano e o PE no que diz respeito às restrições de animacidade a que os sujeitos pronominais plenos estão sujeitos - com uma preferência por antecedentes animados em italiano, independentemente da sua posição estrutural, e uma preferência por antecedentes em posição de objeto em PE, independentemente da sua animacidade (Madeira, Fiéis \& Teixeira, submetido) -, 
não esperamos, de acordo com as predições da HI (Sorace, 2011), problemas persistentes neste domínio, por se tratar de uma propriedade na interface sintaxe-semântica, embora se possam verificar dificuldades em etapas de desenvolvimento. Assim, relativamente à QI.1, predizemos que os aprendentes quase nativos, mas não necessariamente os aprendentes em etapas mais precoces de desenvolvimento, terão um desempenho semelhante ao dos falantes nativos.

Quanto à QI.2, tendo em conta, por um lado, as predições da HI, segundo a qual fenómenos de interfaces externas (como é o caso da interpretação de sujeitos pronominais plenos, que envolve a interface sintaxediscurso) são particularmente vulneráveis na aquisição de L2, e, por outro lado, os resultados de estudos anteriores que corroboram esta predição, mostrando que os aprendentes de L2 tendem a exibir dificuldades persistentes sobretudo na interpretação de sujeitos pronominais plenos (e.g., Lozano, 2003; Sorace, 2016; Sorace \& Filiaci, 2006), prediz-se que aprendentes de italiano L1 - PE L2 exibam opcionalidade permanente na resolução de sujeitos pronominais plenos. Quanto aos sujeitos nulos, apesar de a sua interpretação ser diferente nas duas línguas, espera-se que não haja opcionalidade permanente na sua resolução em PE L2.

\section{Metodologia}

\subsection{Participantes}

Participaram neste estudo 15 falantes nativos de PE e 30 falantes nativos de italiano, com os seguintes níveis de proficiência em PE L2: intermédio alto $(n=10)$, avançado $(n=10)$ e quase nativo $(n=10)$. Todos viviam em Portugal à data do estudo. Os falantes nativos de PE tinham uma média de idade de 25.2 anos. Eram filhos de falantes monolingues de PE, tendo residido durante toda a sua vida em Portugal e tendo o PE como a sua única L1. Os falantes de italiano tinham uma média de idade de 31.4 anos. Todos eram filhos de falantes monolingues de italiano e tinham apenas esta língua como L1. Começaram a ser expostos ao PE entre os 10 e os 38 anos, sendo, portanto, aprendentes adultos de PE L2. Os detalhes sobre o seu perfil sociolinguístico são apresentados na Tabela 1 .

\begin{tabular}{lcccccc}
\hline \multirow{2}{*}{ Grupo } & \multicolumn{2}{c}{ Idade } & \multicolumn{2}{c}{ Idade de início de } & \multicolumn{2}{c}{$\begin{array}{c}\text { Anos de residência em } \\
\text { países lusófonos }\end{array}$} \\
& Média & Desvio padrão regular ao PE & Média & Desvio padrão & Média & Desvio padrão \\
\hline Quase nativo & 37.9 & 7.1 & 28.6 & 6.6 & 8.6 & 3.8 \\
Avançado & 24.2 & 3.3 & 20.1 & 4.6 & 2 & 3.1 \\
Intermédio alto & 32.8 & 11.5 & 23.9 & 5.2 & 4.5 & 7.1 \\
\hline
\end{tabular}

Tabela 1: Dados sobre os aprendentes italianos de PE L2

O nível de proficiência dos falantes de PE L2 foi avaliado através de uma versão adaptada do procedimento de seleção usado por Sorace e Filiaci (2006), originalmente desenhado por White e Genesee (1996) para identificarem falantes quase nativos. No âmbito desta avaliação, todos os participantes foram entrevistados individualmente em PE durante cerca de 5 minutos. Na entrevista, foram usados cartoons (cf. Figura 1) para induzir produção oral espontânea sobre um leque variado de tópicos. O participante respondia a duas perguntas sobre cada cartoon: o que vê no cartoon? Qual é a mensagem do cartoon? 


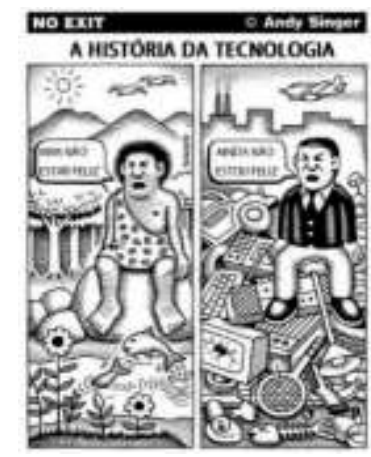

Figura 1: Exemplo de cartoon

(traduzido de https://politicalcartoons.com/sku/16263)

Foi, posteriormente, selecionada uma amostra da produção de cada participante (com cerca de 1.5 minuto) para ser avaliada por três falantes nativos de PE, com base nos seguintes critérios: morfologia, sintaxe, vocabulário, pronúncia, fluência e impressão geral. Na grelha de avaliação fornecida aos avaliadores, cada critério era acompanhado de uma linha contínua de $9 \mathrm{~cm}$, com a designação "não nativo" na ponta esquerda e "nativo" na ponta direita, devendo o avaliador assinalar, com uma cruz, o grau de proximidade do excerto ao nível nativo. Para se assegurar que a ponta nativa da escala era interpretada tendo como ponto de referência falantes nativos de PE, foram misturados, de modo aleatório, excertos de entrevistas com falantes nativos de PE com os excertos das entrevistas dos aprendentes de L2. Após a conclusão de todas as avaliações, os registos dos avaliadores foram transformados em valores discretos, através da sobreposição de um acetato com uma linha de $9 \mathrm{~cm}$ dividida numa escala de 18 pontos (1 ponto por cada $0.5 \mathrm{~cm}$ ).

O nível de proficiência do aprendente foi apurado com base na média de pontos atribuídos pelos avaliadores. Os falantes de PE L2 a quem os avaliadores atribuíram entre 17 e 18 pontos nos critérios sintaxe, morfologia e vocabulário e 15 ou mais pontos nos restantes critérios, ${ }^{2}$ com o máximo de uma exceção, ${ }^{3}$ foram considerados quase nativos. Aqueles que não obtiveram pontuação para serem classificados como quase nativos e receberam, pelo menos, 15 pontos nos critérios sintaxe, morfologia e vocabulário e 12 ou mais pontos nos outros critérios, com o máximo de uma exceção, foram classificados como avançados. Por fim, os aprendentes que não tinham pontos suficientes para serem classificados como avançados e obtiveram, pelo menos, 13 pontos nos critérios sintaxe, morfologia e vocabulário e 10 ou mais pontos nos restantes critérios, com o máximo de uma exceção, foram considerados intermédios altos.

\subsection{Desenho experimental}

Foram utilizadas duas tarefas de escolha múltipla (com e sem pressão de tempo) para elicitar a interpretação preferencial dos participantes em frases complexas em que a oração matriz é seguida de uma oração subordinada adverbial introduzida por quando. As tarefas foram administradas por ordem aleatória em duas ocasiões diferentes, separadas por um intervalo de uma semana. As tarefas tinham um desenho $2 \times 2$, cruzando as variáveis animacidade do objeto da oração matriz (animado vs. não animado) e tipo de sujeito pronominal da oração subordinada (pleno vs. nulo). Ambas as tarefas incluíam 6 itens por condição, num total

\footnotetext{
${ }^{2}$ Esta pontuação é semelhante à atribuída aos falantes nativos, que, ocasionalmente, receberam avaliações de 15 em critérios como fluência.

${ }^{3}$ As exceções são, tipicamente, no critério pronúncia.
} 
de 24 itens experimentais, e 24 distratores. Em todos os itens experimentais, os verbos estavam flexionados no pretérito perfeito do indicativo. Os verbos da oração matriz eram transitivos e os da subordinada inacusativos. Apresentamos itens de exemplo na Tabela 2.

\begin{tabular}{|c|c|c|}
\hline \multirow{2}{*}{$\begin{array}{l}\text { Animacidade } \\
\text { do objeto }\end{array}$} & \multicolumn{2}{|c|}{ Tipo de sujeito pronominal } \\
\hline & Pleno & Nulo \\
\hline [+animado] & $\begin{array}{l}\text { O piloto visitou o colega quando ele chegou } \\
\text { de França. }\end{array}$ & $\begin{array}{l}\text { O piloto visitou o colega quando [-] chegou } \\
\text { de França. }\end{array}$ \\
\hline [-animado] & $\begin{array}{l}\text { O político visitou o navio quando ele chegou } \\
\text { do Brasil. }\end{array}$ & $\begin{array}{l}\text { O político visitou o navio quando [-] chegou } \\
\text { do Brasil. }\end{array}$ \\
\hline
\end{tabular}

Tabela 2: Itens de exemplo

Na tarefa de escolha múltipla sem pressão de tempo, o participante lia uma frase e, com base na sua interpretação preferencial da frase, selecionava a opção mais adequada para completar uma afirmação como a apresentada no exemplo (3). As opções eram as seguintes: o sujeito da matriz, o objeto da matriz, e nem um nem outro. A sua ordem de apresentação era aleatória.

(3) Exemplo de item de teste para a condição sujeito pleno + objeto não animado

O político visitou o navio quando ele chegou do Brasil. chegou do Brasil.

Opções: o político; o navio; nem o político nem o navio

$\mathrm{Na}$ tarefa de escolha múltipla com pressão de tempo, adaptámos o procedimento geralmente usado em tarefas de juízos de aceitabilidade rápidos (e.g., Bader \& Häussler, 2010; Hopp, 2007), para recolhermos dados de interpretação. Em cada item desta tarefa, primeiro, aparecia um ponto de fixação durante $1500 \mathrm{~ms}$ e, em seguida, a frase era apresentada no centro do ecrã palavra por palavra, de forma não cumulativa, a um ritmo de $450 \mathrm{~ms}$ por palavra, tal como é habitual em tarefas de juízos de aceitabilidade rápidos. ${ }^{4}$ Após a última palavra, em vez de se solicitar ao participante que fizesse um juízo de aceitabilidade, pedia-se que completasse uma afirmação sobre a frase que tinha acabado de ler, selecionando uma das três opções fornecidas (ver exemplo 3). Era pedido ao participante que respondesse o mais rapidamente possível. Para cada item, foram gravados o tempo de resposta e a resposta do participante.

As duas tarefas usadas complementam-se. A tarefa de escolha múltipla sem pressão de tempo permite determinar se os aprendentes convergem com a língua alvo a nível representacional. ${ }^{5}$ Por seu lado, a tarefa de escolha múltipla com pressão de tempo permite recolher informação sobre processamento. Vários estudos (para uma síntese, cf. Hopp, 2007) têm mostrado que tarefas offline com pressão de tempo como esta conseguem captar informação sobre processamento, por um lado, porque registam o tempo de resposta do participante, que é considerado um indicador de esforço de processamento (tempos mais longos refletem mais esforço), e, por outro, porque impõem um ritmo de tal forma rápido que forçam o parser a seguir o seu percurso de

\footnotetext{
4400 - 450 ms é o ritmo tipicamente usado neste tipo de tarefas, uma vez que se assume que é uma janela de tempo suficiente para os falantes completarem todos os processos de compreensão normais (cf. Bader \& Häussler, 2010; Hopp, 2007).

${ }^{5}$ Note-se que, como os fenómenos investigados neste estudo não são tipicamente alvo de ensino explícito nas aulas e materiais de PE L2, é altamente improvável que os participantes tivessem conhecimento explícito sobre o que estava a ser testado e o usassem na tarefa sem pressão de tempo, pelo que os resultados desta tarefa deverão refletir o conhecimento implícito dos participantes. Assim, se um participante tiver um desempenho alvo na tarefa sem pressão de tempo e não alvo na tarefa com pressão de tempo, podemos excluir a possibilidade de esta diferença se dever ao facto de a primeira tarefa dar tempo ilimitado ao participante para aceder e usar o seu conhecimento explícito. A causa mais plausível da diferença será o facto de a tarefa com pressão de tempo ser mais sensível a ineficiências de processamento.
} 
processamento preferencial e não dão tempo suficiente para reanálises das frases. Crucialmente, no domínio de aquisição de L2, tem sido proposto que, por serem exigentes em termos de processamento, as tarefas offline com pressão de tempo estão entre as que captam melhor opcionalidade em interfaces linguísticas (cf. Sorace, 2011), conseguindo detetar problemas que tarefas offline sem pressão de tempo não conseguem captar (para evidência a favor desta proposta, ver Teixeira, 2020). A tarefa de escolha múltipla com pressão de tempo é, assim, particularmente adequada para testarmos as predições da HI sobre resolução anafórica em L2.

\subsection{Análise dos dados}

A análise estatística foi realizada usando modelos de efeitos mistos com efeitos aleatórios para participantes e itens. Foram realizadas análises globais dos resultados de cada grupo de participantes, em que foram computados como efeitos fixos as variáveis tipo de sujeito e tipo de objeto. Seguindo Cunnings (2012) e Linck e Cunnings (2015), estas análises incluíram interceções aleatórias para participantes e itens e declives aleatórios por participante para as variáveis tipo de sujeito e tipo de objeto e a sua interação. Além destas análises globais, foram realizados dois tipos de análises adicionais: (i) análises que tinham como objetivo determinar se a diferença entre a escolha de antecedentes sujeito e objeto era significativa numa dada condição experimental (e.g., sujeito nulo + objeto animado, no grupo quase nativo), e (ii) análises que visavam determinar se havia diferenças estatisticamente relevantes na resolução de sujeitos plenos nas condições de objeto animado e de objeto não animado. No primeiro caso, considerámos como efeito fixo a variável antecedente (sujeito vs. objeto) e, no segundo, o efeito fixo era a animacidade do objeto (animado vs. não animado). Estas análises adicionais incluíram interceções aleatórias para participantes e itens e declives aleatórios por participante para a variável antecedente (análise i) ou para a variável animacidade (análise ii).

A análise estatística foi realizada em $\mathrm{R}$, usando o pacote lme4. Mais especificamente, foram usadas a função lmer (modelo misto linear), para a análise dos tempos de resposta da tarefa com pressão de tempo, e a função glmer (modelo misto linear generalizado), com a especificação "family=binomial", para a análise das respostas nas tarefas com e sem pressão de tempo. Como a taxa de seleção da opção "nem antecedente sujeito nem antecedente objeto" foi baixa, variando entre $0 \%$ e $20 \%$, as respostas foram tratadas como binárias e codificadas como 'antecedente sujeito' $=1$ e 'antecedente não sujeito' $=0$. Nos casos em que se pretendia contrastar a escolha de objeto e de sujeito (i.e., análise i), computámos o antecedente como um efeito fixo com dois níveis, objeto e sujeito, e, em cada nível, as respostas foram codificadas como 'sim' = 1 e 'não' =0. Em todas as análises, os efeitos fixos foram codificados contrastivamente com os códigos 0.5 e -0.5 para comparar dois níveis do mesmo efeito fixo (e.g., objeto animado $=0.5$ vs. objeto não animado $=-0.5$ ).

\section{Resultados}

\subsection{Tarefa sem pressão de tempo}

Na tarefa de escolha múltipla sem pressão de tempo, observa-se, no grupo de PE L1, um efeito de tipo de sujeito pronominal (estimativa $=-7.2673, \mathrm{EP}=1.5180, p<.001$ ), mas nenhum efeito de animacidade do objeto nem nenhuma interação entre este fator e o tipo de sujeito ( $p s \geq .299$ ). Como mostram as Figuras 2 e 3, em PE, os sujeitos pronominais nulos retomam antecedentes em posição de sujeito e os plenos retomam antecedentes em posição de objeto, independentemente da animacidade do objeto (cf. Tabela 3). No nível quase nativo, os aprendentes de PE L2 têm um desempenho idêntico ao dos falantes de PE L1 quer em relação aos sujeitos nulos, quer em relação aos sujeitos plenos (cf. Figuras 2 e 3 e Tabelas 3 e 4). Já nos níveis avançado e intermédio, os aprendentes têm um desempenho em muitos aspetos diferente do dos falantes de PE L1. Como se pode observar na Figura 2 e na Tabela 3, ao contrário destes falantes, os aprendentes avançados e intermédios exibem efeitos de animacidade na interpretação de sujeitos pronominais plenos. Quando o objeto é animado, os dois grupos de 
aprendentes interpretam o sujeito pleno como correferente com o antecedente na posição de objeto, tal como os falantes de PE L1. Contudo, quando o objeto não é animado, esta preferência desaparece: no grupo avançado, há opcionalidade entre os antecedentes sujeito e objeto e, no grupo intermédio, o sujeito pleno tende a retomar o antecedente na posição de sujeito (cf. Figura 2 e Tabela 4). Ambos os grupos divergem, assim, dos falantes de PE L1 na condição sujeito pleno + objeto não animado. Na resolução de sujeitos nulos, o grupo avançado, tal como os falantes PE L1, tem uma preferência clara pela retoma do antecedente na posição de sujeito da oração matriz, quando o objeto não é animado. No entanto, nos casos em que o objeto é animado, a diferença na escolha dos antecedentes sujeito e objeto não chega a ser significativa, apesar de se aproximar do limiar de significância estatística (cf. Tabela 4). O grupo intermédio, por seu lado, exibe sempre opcionalidade entre os antecedentes sujeito e objeto na resolução de sujeitos nulos (cf. Figura 3 e Tabela 4), divergindo, assim, claramente dos falantes de PE L1.

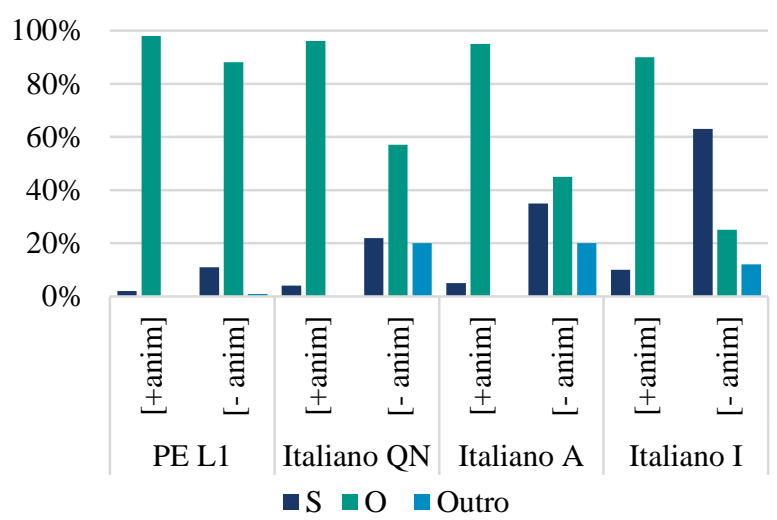

Figura 2: Interpretação do sujeito pleno na tarefa sem pressão de tempo por tipo de objeto

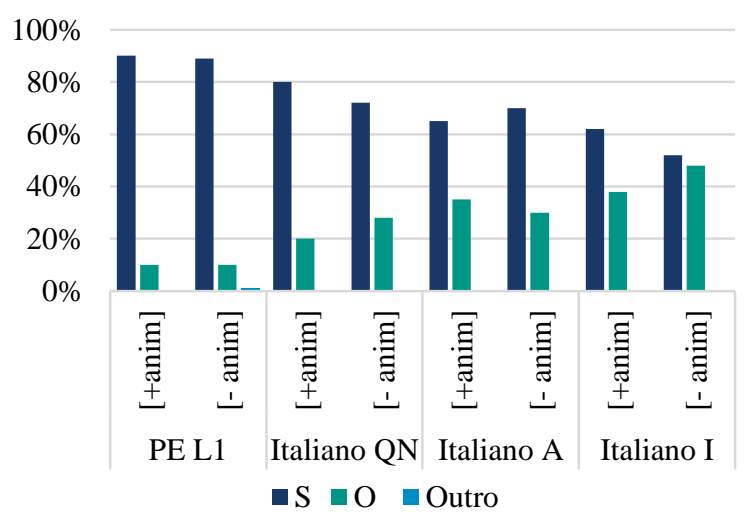

Figura 3: Interpretação do sujeito nulo na tarefa sem pressão de tempo por tipo de objeto

\begin{tabular}{cccc}
\hline Grupo & Estimativa & Erro Padrão & $\boldsymbol{p}$ \\
\hline PE L1 & 9.231 & 18.147 & .611 \\
Italiano QN & -5.780 & 4.050 & .1536 \\
Italiano A & 2.5417 & .8930 & $.004425^{*}$ \\
Italiano I & 3.3389 & .6784 & $<.001^{*}$ \\
\hline
\end{tabular}

Legenda: * = estatisticamente significativo

Tabela 3: Diferença entre as condições S pleno + O animado e S pleno + O não animado na tarefa sem pressão de tempo

\begin{tabular}{clccc}
\hline Grupo & \multicolumn{1}{c}{ Condição } & Estimativa & Erro Padrão & $\boldsymbol{P}$ \\
\hline PE L1 & S pleno + O animado & 5.7514 & 1.2552 & $.00149^{*}$ \\
& S pleno + O não animado & 5.8626 & 1.2672 & $<.001^{*}$ \\
& S nulo + O animado & -4.8066 & .7499 & $<.001^{*}$ \\
& S nulo + O não animado & -10.533 & 4.011 & $.00864^{*}$ \\
\hline \multirow{2}{*}{ Italiano QN } & S pleno + O animado & -6.0913 & .9339 & $<.001^{*}$ \\
& S pleno + O não animado & -2.1855 & 1.1741 & $.0427^{*}$ \\
& S nulo + O animado & 4.144 & 1.570 & $.00828^{*}$
\end{tabular}




\begin{tabular}{clccc} 
& S nulo + O não animado & 2.7222 & 1.1475 & $.0177^{*}$ \\
\hline Italiano A & S pleno + O animado & -6.200 & 1.318 & $<.001^{*}$ \\
& S pleno + O não animado & -0.8411 & 1.5361 & .584 \\
& S nulo + O animado & 1.9494 & 1.1506 & .0902. \\
& S nulo + O não animado & 2.17094 & .88688 & $.0144^{*}$ \\
\hline Italiano I & S pleno + O animado & 5.013 & 1.096 & $<.001^{*}$ \\
& S pleno + O não animado & -2.2412 & .9763 & $.0217^{*}$ \\
& S nulo + O animado & -1.478 & 1.118 & .186 \\
& S nulo + O não animado & -4.026 & 9.840 & .482 \\
\hline
\end{tabular}

Legenda: $*$ = estatisticamente significativo; . = próximo de significância estatística

Tabela 4: Diferença entre escolha de antecedente sujeito e objeto por condição e grupo na tarefa sem pressão de tempo

\subsection{Tarefa com pressão de tempo}

Na tarefa com pressão de tempo, os falantes de PE L1 têm um desempenho idêntico ao exibido na tarefa sem pressão de tempo, observando-se apenas um efeito de tipo de sujeito pronominal na análise estatística dos seus dados (estimativa $=-5.53164, \mathrm{EP}=1.09437, p<.001$ ). Nenhum outro efeito ou interação é significativo ( $p \mathrm{~s} \geq .462$ ). Como as Figuras 4 e 5 mostram, mesmo sob pressão de tempo, os falantes de PE L1 continuam a interpretar o sujeito nulo como correferente com o sujeito da oração matriz e o sujeito pleno como correferente com o objeto, independentemente de o objeto ser ou não animado (cf. Tabela 5). Ao contrário destes falantes, os falantes quase nativos de PE têm um desempenho diferente quando estão com e sem pressão de tempo. Enquanto, na tarefa sem pressão de tempo, convergem com o grupo de controlo em todas as condições, na tarefa com pressão de tempo, tal só acontece na condição sujeito pleno + objeto animado. Em todas as outras condições, exibem opcionalidade entre a retoma do antecedente sujeito e a retoma do antecedente na posição de objeto (cf. Tabela 6), divergindo, deste modo, dos falantes de PE L1. Contrariamente ao grupo quase nativo, os restantes grupos de aprendentes de PE L2 têm um desempenho semelhante nas tarefas com e sem pressão de tempo. Só há diferenças entre as duas tarefas na interpretação dos sujeitos nulos por parte do grupo avançado. Como vimos, na tarefa sem pressão de tempo, o grupo avançado tem um desempenho alvo na interpretação de sujeitos nulos, quando o objeto é não animado, e apresenta uma preferência quase significativa pelo sujeito, quando o objeto é animado. Já na tarefa de escolha múltipla com pressão de tempo, este grupo exibe sempre opcionalidade na resolução de sujeitos nulos, independentemente da animacidade do objeto (cf. Figura 5 e Tabela 6). Relativamente aos tempos de resposta, não foram observadas diferenças relevantes nos dados de cada grupo de aprendentes, pelo que não os incluímos no presente artigo. 


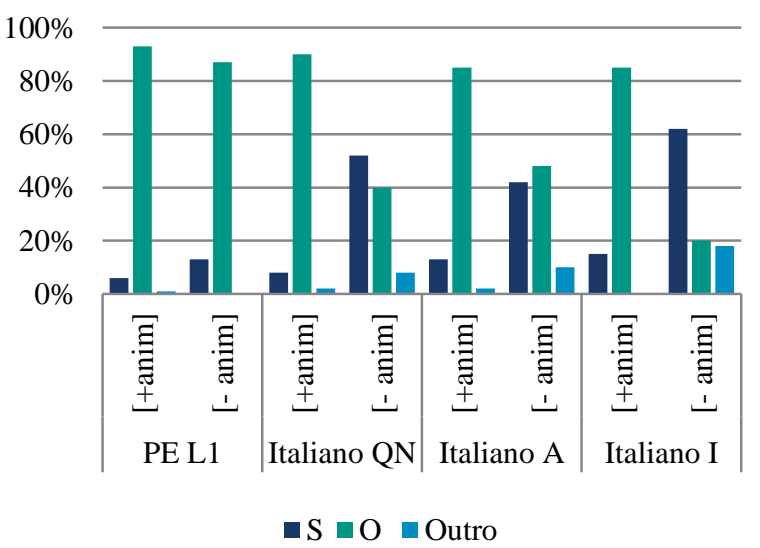

Figura 4: Interpretação do sujeito pleno na tarefa com pressão de tempo por tipo de objeto

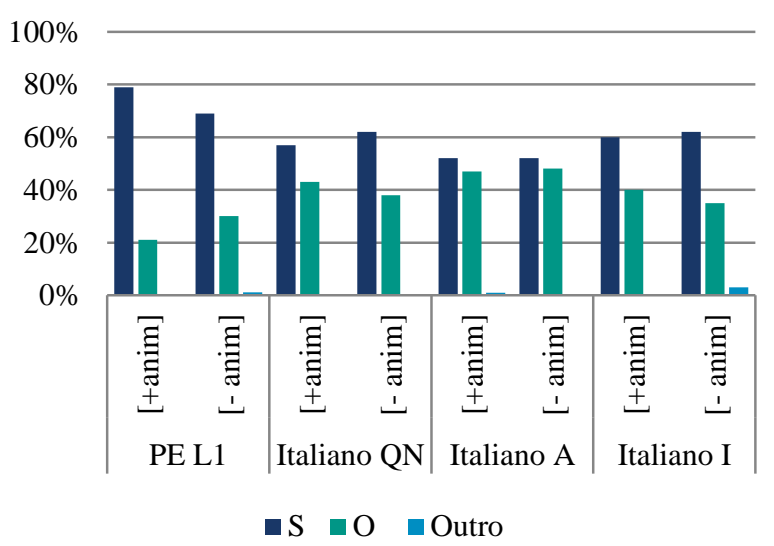

Figura 5: Interpretação do sujeito nulo na tarefa com pressão de tempo por tipo de objeto

\begin{tabular}{cccc}
\hline Grupo & Estimativa & Erro Padrão & $\boldsymbol{p}$ \\
\hline PE L1 & .5581 & 1.4797 & .706024 \\
Italiano QN & 3.8435 & 1.4989 & $.0103^{*}$ \\
Italiano A & 2.1717 & 1.1085 & $.0401^{*}$ \\
Italiano I & 2.6453 & .8503 & $.00186^{*}$ \\
\hline
\end{tabular}

Legenda: * = estatisticamente significativo

Tabela 5: Diferença entre as condições $\mathrm{S}$ pleno + $\mathrm{O}$ animado e $\mathrm{S}$ pleno + $\mathrm{O}$ não animado na tarefa com pressão de tempo

\begin{tabular}{clccc}
\hline Grupo & \multicolumn{1}{c}{ Condição } & Estimativa & Erro Padrão & $\boldsymbol{P}$ \\
\hline PE L1 & S pleno + O animado & 6.7023 & 1.5419 & $<.001^{*}$ \\
& S pleno + O não animado & 4.2415 & .7455 & $<.001^{*}$ \\
& S nulo + O animado & -3.1900 & .6094 & $<.001^{*}$ \\
& S nulo + O não animado & -2.1827 & .6137 & $<.001^{*}$ \\
\hline \multirow{2}{*}{ Italiano QN } & S pleno + O animado & -5.7983 & 1.6379 & $<.001^{*}$ \\
& S pleno + O não animado & .5154 & 1.6245 & .751 \\
& S nulo + O animado & 1.717 & 0.9380 & .397 \\
& S nulo + O não animado & 1.23031 & .83025 & .138 \\
\hline Italiano A & S pleno + O animado & 5.26621 & 1.61076 & .586 \\
& S pleno + O não animado & .5690 & 1.0457 & .706 \\
& S nulo + O animado & -.39924 & 1.05678 & .743 \\
\hline
\end{tabular}




\begin{tabular}{lccc} 
S pleno + O não animado & -3.0240 & 1.3460 & $.0247^{*}$ \\
S nulo + O animado & -1.0410 & 1.037 & .315 \\
S nulo + O não animado & -1.66707 & 1.03676 & .108 \\
\hline
\end{tabular}

Legenda: * = estatisticamente significativo

Tabela 6: Diferença entre escolha do antecedente sujeito e objeto por condição e grupo na tarefa com pressão de tempo

\subsection{Sumário}

Em suma, os resultados do presente estudo mostram que, em PE L1, os sujeitos plenos retomam preferencialmente antecedentes objeto e os sujeitos nulos retomam antecedentes sujeito, independentemente dos traços de animacidade do objeto. Na interpretação de sujeitos plenos, os aprendentes de PE L2 convergem com o grupo de PE L1 em ambas as tarefas e em todos os níveis de proficiência, quando o objeto é animado. Já quando não é animado, todos os grupos de aprendentes divergem do grupo de controlo, exceto o grupo quase nativo na tarefa sem pressão de tempo. Os aprendentes exibem, assim, efeitos de animacidade na interpretação de sujeitos pronominais plenos em PE L2. Na resolução de sujeitos nulos, há opcionalidade em todos os grupos de aprendentes menos nos grupos quase nativo e avançado na tarefa sem pressão de tempo. Os resultados dos falantes de PE L1 e dos aprendentes de PE L2 são sintetizados na Tabela 7.

\begin{tabular}{|c|c|c|c|c|c|c|}
\hline \multirow[t]{2}{*}{ Tarefa } & \multirow{2}{*}{$\begin{array}{l}\text { Tipo de } \\
\text { sujeito }\end{array}$} & \multirow{2}{*}{$\begin{array}{c}\text { Animacidade do } \\
\text { objeto }\end{array}$} & \multicolumn{4}{|c|}{ Grupo } \\
\hline & & & $P E L I$ & Italiano $Q N$ & Italiano $A$ & Italiano I \\
\hline \multirow{4}{*}{$\begin{array}{l}\text { Sem pressão } \\
\text { de tempo }\end{array}$} & Pleno & [+ animado] & $\mathrm{O}$ & $\mathrm{O}$ & $\mathrm{O}$ & $\mathrm{O}$ \\
\hline & & [- animado] & $\mathrm{O}$ & $\mathrm{O}$ & $\mathrm{S} / \mathrm{O}$ & $\mathrm{S}$ \\
\hline & Nulo & [+ animado] & $S$ & $S$ & $\mathrm{~S}^{\mathrm{a}}$ & $\mathrm{S} / \mathrm{O}$ \\
\hline & & [- animado] & $\mathrm{S}$ & $S$ & $\mathrm{~S}$ & $\mathrm{~S} / \mathrm{O}$ \\
\hline \multirow{4}{*}{$\begin{array}{l}\text { Com pressão } \\
\text { de tempo }\end{array}$} & Pleno & [+ animado] & $\mathrm{O}$ & $\mathrm{O}$ & $\mathrm{O}$ & $\mathrm{O}$ \\
\hline & & [- animado] & $\mathrm{O}$ & $\mathrm{S} / \mathrm{O}$ & $\mathrm{S} / \mathrm{O}$ & $S$ \\
\hline & Nulo & [+ animado] & $S$ & $\mathrm{~S} / \mathrm{O}$ & $\mathrm{S} / \mathrm{O}$ & $\mathrm{S} / \mathrm{O}$ \\
\hline & & [- animado] & S & $\mathrm{S} / \mathrm{O}$ & $\mathrm{S} / \mathrm{O}$ & $\mathrm{S} / \mathrm{O}$ \\
\hline
\end{tabular}

Nota: Recorde-se que, neste caso, a preferência pelo sujeito é quase significativa.

Tabela 7: Preferências interpretativas em PE L1 e L2 por tarefa e condição

\section{Discussão e conclusões}

Retomamos aqui as duas questões de investigação que estiveram na base deste estudo, procurando compreender que respostas os resultados acima descritos sugerem para estas questões.

QI.1 - Na resolução de sujeitos pronominais plenos, os aprendentes de italiano L1 - PE L2 têm um desempenho semelhante ao dos falantes nativos de PE no que diz respeito à animacidade do antecedente em diferentes estádios de desenvolvimento? 
Os resultados mostram que as propriedades na interface sintaxe-semântica, como é o caso do efeito da animacidade na resolução anafórica de sujeitos pronominais plenos, podem ser persistentemente problemáticas numa L2, contrariando a nossa predição de que os aprendentes quase nativos teriam um desempenho semelhante aos falantes nativos. De facto, observou-se que, no nível quase nativo, os aprendentes italianos de PE apresentam um desempenho alvo na interpretação dos sujeitos plenos, mas apenas na tarefa sem pressão de tempo. Na tarefa com pressão de tempo, estes aprendentes apresentam opcionalidade na condição em que o antecedente objeto é não animado.

Embora os aprendentes italianos de PE L2 não atinjam um desempenho nativo na condição de sujeito pleno + antecedente objeto não animado, observa-se um efeito de desenvolvimento nesta condição em ambas as tarefas. Os aprendentes de nível intermédio mostram preferência pela retoma de um antecedente em posição de sujeito, o que corresponde à preferência da sua L1 (cf. Madeira, Fiéis \& Teixeira, submetido). No nível avançado, esta preferência é abandonada, observando-se opcionalidade na seleção do antecedente. Finalmente, os aprendentes quase-nativos apresentam, como vimos, preferência por um antecedente objeto, à semelhança dos falantes nativos de PE, perdendo-se, no entanto, esta preferência sob pressão de tempo.

Estes resultados colocam em causa a proposta da $\mathrm{HI}$ de que as propriedades de interfaces internas, como é o caso da interface sintaxe-semântica, não são persistentemente problemáticas. As diferenças nos resultados dos aprendentes quase nativos nas duas tarefas e, em particular, a constatação de que estes aprendentes apresentam um desempenho alvo na tarefa sem pressão de tempo sugerem que a opcionalidade exibida na tarefa com pressão de tempo se deve a ineficiências de processamento e não a défices representacionais. Este facto indica que o conhecimento destas propriedades é plenamente adquirível em L2.

Ao contrário do que tem sido proposto em estudos anteriores (e.g., Sorace \& Filiaci, 2006; Sorace, 2016), os nossos resultados apresentam fortes indícios da presença de um efeito de influência da L1 na resolução de sujeitos pronominais plenos. Este efeito é muito visível no desempenho dos aprendentes de nível intermédio, que apresentam evidências de transferência para PE L2 das restrições de animacidade que condicionam a interpretação de sujeitos pronominais plenos na sua L1, o italiano.

QI.2 - Quando o antecedente na posição de objeto é animado, os aprendentes de italiano L1 - PE L2 exibem opcionalidade permanente na interpretação de pronomes plenos, mas não na de nulos, conforme proposto por Sorace (2016), e.o.?

Os resultados mostram não haver dificuldades na resolução de sujeitos pronominais plenos em L2 em nenhuma das duas tarefas quando o antecedente na posição de objeto é animado, exibindo os aprendentes italianos de PE L2 uma preferência sistemática pela retoma do antecedente objeto, à semelhança dos falantes nativos de PE. Verificamos que é na resolução de sujeitos nulos que os aprendentes manifestam dificuldades. Embora na tarefa sem pressão de tempo os aprendentes quase nativos apresentem um desempenho alvo, com preferência pelo antecedente sujeito, esta preferência desaparece na tarefa com pressão de tempo, em que se observa opcionalidade. Encontra-se opcionalidade também no nível intermédio nas duas tarefas e no grupo avançado na tarefa com pressão de tempo, que poderá resultar de transferência da L1, uma vez que este é o padrão identificado na interpretação de sujeitos nulos em italiano (cf. Madeira, Fiéis \& Teixeira, submetido; Sorace \& Filiaci, 2006). Estes resultados contrariam a nossa predição de que os aprendentes de italiano L1 - PE L2 apresentariam opcionalidade permanente na interpretação de sujeitos pronominais plenos, mas não na de sujeitos nulos, e constituem evidência contra a proposta da HI de que, na resolução anafórica em L2, os sujeitos plenos são problemáticos e os sujeitos nulos são fáceis.

$\mathrm{Na}$ interpretação de sujeitos nulos, observa-se, no desempenho dos aprendentes quase nativos, um padrão semelhante ao verificado relativamente ao efeito de animacidade na interpretação de sujeitos plenos, com uma assimetria entre as duas tarefas. $\mathrm{O}$ facto de que estes aprendentes exibem um desempenho alvo na tarefa sem 
pressão de tempo confirma a nossa conclusão de que a opcionalidade exibida na tarefa com pressão de tempo pode ser atribuída a ineficiências de processamento e não a problemas representacionais, sendo as propriedades interpretativas dos sujeitos nulos plenamente adquiríveis em L2.

Em suma, os resultados dos aprendentes parecem ser determinados não pelos tipos de interface que cada fenómeno envolve, mas sim pelas diferenças entre a L1 e a L2. As áreas em que os aprendentes de italiano L1 - PE L2 apresentam um comportamento divergente do grupo de controlo no presente estudo são precisamente aquelas em que existe microvariação no italiano e no PE. Como referido na secção 2.1, estas duas línguas diferem quer em relação à resolução de sujeitos nulos, que se caracteriza por uma tendência mais forte para retoma de antecedentes sujeito em PE do que em italiano, quer na resolução de sujeitos plenos com um antecedente objeto não animado, em que se observa um efeito de animacidade mais forte em italiano do que em PE. O presente estudo mostra que os aprendentes se vão tornando progressivamente sensíveis à microvariação à medida que o seu nível de proficiência aumenta. Contudo, o seu desempenho permanece permanentemente instável nas áreas em que há diferenças entre a L1 e a L2. Estes resultados apontam, pois, para a importância da influência da L1 na resolução anafórica em L2, um fator que, como vimos na secção 2.2, tem sido minimizado em trabalhos anteriores. Também a proposta de que apenas os sujeitos plenos são problemáticos no estádio final de aquisição de L2 é colocada em questão pelos resultados do presente trabalho.

\section{Referências}

Alonso-Ovalle, Luis, Susana Fernández-Solera, Lyn Frazier \& Charles Clifton (2002) Null vs. overt pronouns and the topic-focus articulation in Spanish. Journal of Italian Linguistics 14, pp. 151-169.

Bader, Markus \& Jana Häussler (2010) Toward a model of grammaticality judgments. Journal of Linguistics 46 (2), pp. 273-330.

Barbosa, Pilar, Maria Eugênia Duarte \& Mary Kato (2005) Null subjects in European and Brazilian Portuguese. Journal of Portuguese Linguistics 4 (2), pp. 11-52.

Belletti, Adriana, Elisa Bennati \& Antonella Sorace (2007) Theoretical and developmental issues in the syntax of subjects: Evidence from near-native Italian. Natural Language \& Linguistic Theory 25 (4), pp. 657-689.

Bini, Milena (1993) La adquisicíon del italiano: Mas allá de las propiedades sintácticas del parámetro pro-drop. In. Juana Liceras (Ed.) La linguistica y el analisis de los sistemas no nativos. Ottawa: Doverhouse, pp. 126139.

Cardinaletti, Anna \& Michal Starke (1999) The typology of structural deficiency: A case study of the three classes of pronouns. In. Henk van Riemsdijk (Ed.) Clitics in the languages of Europe. Berlin/New York: Mouton de Gruyter, pp. 145-233.

Carminati, Maria Nella (2002) The processing of Italian subject pronouns. Dissertação de doutoramento, University of Massachusetts.

Cunnings, Ian (2012) An overview of mixed-effects statistical models for second language researchers. Second Language Research 28 (3), pp. 369-382.

Filiaci, Francesca (2010) Null and overt subject biases in Spanish and Italian: A cross-linguistic comparison. In. Claudia Borgonovo, Manuel Español-Echevarría, \& Philippe Prévost (Eds.) Selected Proceedings of the 12th Hispanic Linguistics Symposium. Somerville, MA: Cascadilla Proceedings Project, pp. 171-182.

Filiaci, Francesca, Antonella Sorace \& Manuel Carreiras (2014) Anaphoric biases of null and overt subjects in Italian and Spanish: a cross-linguistic comparison. Language, Cognition and Neuroscience 29 (7), pp. 825843.

Hopp, Holger (2007) Ultimate attainment at the interfaces in second language acquisition: Grammar and processing. Dissertação de doutoramento, University of Groningen. 
Kraš, Tihana (2011) Acquiring the syntactic constraints on auxiliary change under restructuring in L2 Italian: Implications for the Interface Hypothesis. Linguistic Approaches to Bilingualism 1 (4), pp. 413-438.

Linck, Jared, \& Ian Cunnings (2015) The utility and application of mixed-effects models in second language research. Language Learning 65, pp. 185-207.

Lobo, Maria, Ana Madeira \& Carolina Silva (2017) Interpretação de pronomes sujeito anafóricos e catafóricos por falantes de português L2: efeitos da língua materna. Revista da Associação Portuguesa de Linguística 3, pp. 135-153.

Lozano, Cristóbal (2003) Universal Grammar and focus constraints: The acquisition of pronouns and word order in non-native Spanish. Dissertação de Doutoramento, University of Essex.

Lozano, Cristóbal (2006) Focus and split-intransitivity: The acquisition of word order alternations in non-native Spanish. Second Language Research 22 (2), pp. 145-187.

Lozano, Cristóbal (2008) ¿Déficits de representación o de procesamiento en una segunda lengua? Evidencia de un estudio de resolución de anáfora con griegos adultos aprendices de español. In. Rafael Monroy \& Aquilino Sánchez (Eds.) 25 años de Lingüística Aplicada en España: Hitos y retos / 25 Years of Applied Linguistics in Spain: Milestones and Challenges. Murcia: Editum, pp. 855-866.

Luegi, Paula (2012) Processamento de sujeitos pronominais em Português: efeito da posição estrutural do antecedente. Dissertação de doutoramento, Faculdade de Letras da Universidade de Lisboa.

Madeira, Ana, Alexandra Fiéis \& Joana Teixeira (neste volume) Microvariação na resolução de sujeitos pronominais: português europeu vs. italiano. Revista da Associação Portuguesa de Linguística, 8.

Margaza, Panagiota \& Aurora Bel (2006) Null subjects at the syntax-pragmatics interface: Evidence from Spanish interlanguage of Greek speakers. In. Mary Grantham O’Brien, Christine Shea \& John Archibald (Eds.) Proceedings of GASLA 2006. Somerville, MA: Cascadilla Press, pp. 88-97.

Mendes, Célia \& I. Carolina Iribarren (2007) Fixação do parâmetro do sujeito nulo na aquisição do português europeu por hispanofalantes. In. Maria Lobo \& Maria Antónia Coutinho (Eds.) Textos seleccionados do XXII Encontro Nacional da Associação Portuguesa de Linguística. Lisboa: Associação Portuguesa de Linguística, pp. 483-498.

Montalbetti, Mario (1984) After binding. On the interpretation of pronouns. Dissertação de doutoramento, MIT.

Morgado, Sara, Paula Luegi \& Maria Lobo (2018) Efeitos de animacidade do antecedente na resolução de pronomes sujeito. Revista da Associação Portuguesa de Linguística 4, pp. 190-205.

Rothman, Jason (2009) Pragmatic deficits with syntactic consequences?: L2 pronominal subjects and the syntax-pragmatics interface. Journal of Pragmatics 41 (5), pp. 951-973.

Serratrice, Ludovica, Antonella Sorace, Francesca Filiaci \& Michela Baldo (2009) Bilingual children's sensitivity to specificity and genericity: Evidence from metalinguistic awareness. Bilingualism: Language and Cognition 12 (2), pp. 239-257.

Sorace, Antonella (2011) Pinning down the concept of 'interface' in bilingualism. Linguistic Approaches to Bilingualism 1, pp. 1-33.

Sorace, Antonella (2016) Referring expressions and executive functions in bilingualism. Linguistic Approaches to Bilingualism 6 (5), pp. 669-684.

Sorace, Antonella \& Francesca Filiaci (2006) Anaphora resolution in near-native speakers of Italian. Second Language Research 22 (3), pp. 339-368.

Sorace, Antonella \& Ludovica Serratrice (2009) Internal and external interfaces in bilingual language development: Beyond structural overlap. International Journal of Bilingualism 13 (2), pp. 195-210.

Sorace, Antonella, Ludovica Serratrice, Francesca Filiaci \& Michela Baldo (2009) Discourse conditions on subject pronoun realization: Testing the linguistic intuitions of older bilingual children. Lingua 119 (3), pp. 460-477. 
Teixeira, Joana (2020) Gradient optionality in L2 acquisition at the syntax-discourse interface: Evidence from inversion in advanced and near-native English. Lingua 245,102947.

Tsimpli, Ianthi \& Antonella Sorace (2006) Differentiating interfaces: L2 performance in syntax-semantics and syntax-discourse phenomena. In. David Bamman, Tatiana Magnitskaia \& Colleen Zaller (Eds.) Proceedings of the 30th Annual Boston University Conference on Language Development. Somerville, MA: Cascadilla Press, pp. 653-664.

White, Lydia \& Fred Genesee (1996) How native is near-native? The issue of ultimate attainment in adult second language acquisition. Second Language Research 12 (3), pp. 233-265. 